\title{
The Hartree-Fock Theory for Coulomb Systems
}

\author{
Elliott H. Lieb* \\ Barry Simon $\star \star$ \\ Department of Physics, Yeshiva University, New York, New York 10033, USA
}

Departments of Mathematics and Physics, Princeton University, Princeton, New Jersey 08540, USA

\begin{abstract}
For neutral atoms and molecules and positive ions and radicals, we prove the existence of solutions of the Hartree-Fock equations which minimize the Hartree-Fock energy. We establish some properties of the solutions including exponential falloff.
\end{abstract}

\section{$\S 1$. Introduction}

In this paper we discuss the Hartree $(\mathrm{H})$ and Hartree-Fock (HF) theories associated with the purely Coulombic Hamiltonian of electrons interacting with static nucleii. Our purpose will be to prove that these theories exist (in the sense that the equations have solutions which minimize the $\mathrm{H}$ or HF energy) whenever the system has an excess positive charge after the removal of one electron. An announcement of these results was given in [22] and an outline of the proof was given in [19].

The precise quantum system is described by the Hamiltonian

$$
H=-\sum_{i=1}^{N} \Delta_{i}+\sum_{i=1}^{N} V\left(x_{i}\right)+\sum_{i<j}\left|x_{i}-x_{j}\right|^{-1},
$$

where

$$
V(x)=-\sum_{j=1}^{k} z_{j}\left|x-R_{j}\right|^{-1}
$$

acting on the Hilbert space $\mathscr{H}=L_{a}^{2}\left(\mathbb{R}^{3 N} ; \mathbb{C}^{2 N}\right)$. We assume $z_{j}>0$, all $j$. The subscript $a$ on $L^{2}$ indicates that we are to consider functions in $L^{2}$ as $\Psi\left(x_{1}, \sigma_{1} ; \ldots ; x_{N}, \sigma_{N}\right)$ with $x_{i} \in \mathbb{R}^{3}, \sigma_{i} \in \pm 1 / 2$ and only allow those $\Psi$ antisymmetric under interchanges of $i$ and $j$. The particles have two spin states, but we could allow $q$ spin states in our analysis below with only notational changes. The physically correct Fermi statistics

* Research partially supported by U.S. National Science Foundation Grant MCS-75-21684

$\star \star$ Research partially supported by U.S. National Science Foundation under Grants MPS-75-11864 and MPS-75-20638. On leave from Departments of Mathematics and Physics, Princeton University, Princeton, NJ 08540, USA 
(antisymmetric functions) which we impose turns out to be the most difficult; our method would apply equally well to any other kind of statistics.

In (2), the $z_{j}$ are the charges of the nucleii at positions $R_{j}$. By a famous theorem of Kato [16], $\mathrm{H}$ is essentially self-adjoint on $C_{0}^{\infty}\left(\mathbb{R}^{3 N} ; \mathbb{C}^{2 N}\right)_{a}=\mathscr{D}_{\text {phys }}$, the $C^{\infty}$ functions of compact support.

We set:

$$
E_{N}^{Q}\left(z_{i}, R_{i}\right) \equiv \inf \left\{(\Psi, H \Psi) \mid \Psi \in \mathscr{D}_{\text {phys }} ;\|\Psi\|=1\right\}
$$

which is defined to be the quantum ground state energy.

In 1928, Hartree [14] introduced an approximate method for finding $E_{N}^{Q}$. He apriori ignored the spin variables and the Pauli principle and considered product wave functions.

$$
\Psi\left(x_{1}, \ldots, x_{N}\right)=\prod_{i=1}^{N} u_{i}\left(x_{i}\right) .
$$

Minimization of the functional

$$
\mathscr{E}_{\mathrm{H}}\left(u_{1}, \ldots, u_{N}\right)=(\Psi, H \Psi)
$$

with the constraint $\left\|u_{i}\right\|=1$ then leads to the Euler-Lagrange equation

$$
\tilde{h}_{i} u_{i}=\varepsilon_{i} u_{i},
$$

where the $\varepsilon_{i}$ are Lagrange multipliers and

$$
\begin{aligned}
& \left(\tilde{h}_{i} w\right)(x)=(-\Delta w)(x)+V(x) w(x)+R_{\Psi}^{i}(x) w(x), \\
& R_{\Psi}^{i}(x)=\sum_{j \neq i} \int|x-y|^{-1}\left|u_{j}(y)\right|^{2} d^{3} y .
\end{aligned}
$$

Note that in the $\mathrm{H}$ equations, (5), the $h_{i}$ depend non-trivally on $i$. This is to be contrasted with the HF equations (7) where $h$ is independent of $i$. Of course, the equations (5) formally only correspond to stationary points of $\mathscr{E}_{\mathrm{H}}$ so there should be solutions corresponding to $u$ 's that do not minimize $\mathscr{E}_{\mathrm{H}}$. Hartree attempted to take the Pauli principle into account by seeking solutions with $u_{1}=u_{2}$ and $u_{3}$ "approximately orthogonal to $u_{1}$ ", $u_{3}=u_{4}$ etc. [We should also mention that Hartree's derivation of (5) did not go through a minimization in the variational principle-this is a refinement due to Slater [30] which led him to the HF equations.] A more systematic and satisfactory way to take the Pauli principle into account was discovered in 1930 independently by Fock [10] and Slater [30] yielding equations now called Hartree-Fock (HF) equations. One considers trial functions $u_{i}\left(x_{i}, \sigma_{i}\right) ; i$ $=1, \ldots, N$ with $\left(u_{i}, u_{j}\right)=\delta_{i j}$ and the Slater determinant

$$
\Psi\left(x_{1}, \sigma_{1}, \ldots, x_{N}, \sigma_{N}\right)=(N !)^{-1 / 2} \operatorname{det}\left(u_{i}\left(x_{j}, \sigma_{j}\right)\right)
$$

and minimizes

$$
\mathscr{E}_{\mathrm{HF}}\left(u_{1}, \ldots, u_{N}\right)=(\Psi, H \Psi)
$$


with the constraint $\left(u_{i}, u_{j}\right)=\delta_{i j}$. The corresponding Euler-Lagrange equations are:

$$
\begin{aligned}
& h u_{i}=\varepsilon_{i} u_{i}, \\
& (h w)(x)=(-\Delta w)(x)+V(x) w(x)+U_{\Psi}(x) w(x)-\left(K_{\Psi} w\right)(x), \\
& U_{\Psi}(x)=\sum_{j=1}^{N} \int|x-y|^{-1}\left|u_{j}(y)\right|^{2} d^{3} y, \\
& \left(K_{\Psi} w\right)(x)=\sum_{j=1}^{N} u_{j}(x) \int|x-y|^{-1} \overline{u_{j}(y)} w(y) d^{3} y .
\end{aligned}
$$

$U_{\Psi}$ is the "direct" interaction and $K_{\Psi}$ is the "exchange" interaction. We will show that minimizing solutions of (7a) exist whenever $N<Z+1$ where $Z$ is the nuclear charge

$$
Z=\sum_{j=1}^{k} z_{j}
$$

We make the convention that when $u$ 's depending on spin are involved, as in $(7 \mathrm{c})$ and (7d) the symbol $\int-d^{3} y$ indicates also a sum over the spin variable attached to $y$. [We note that the naive Euler-Lagrange equations are more complicated than (7) but after a unitary change, $u_{i}^{\text {New }}=\sum a_{i j} u_{j}^{\text {Old }}$ with $a_{i j}$ a unitary $N \times N$ matrix, (7) results. The Slater determinant (6a) is unaffected by the change so that (6b) is unaffected. This is proved in Lemma 2.3 and is further discussed in many texts, e.g. Bethe-Jackiw [6]; it plays an important role in $\S 2$ below.]

Irrespective of the physical content of the $\mathrm{H}$ and HF equations, (5), (7), it is far from evident that there exist any solutions of them, let alone minimizing solutions, for they are clearly complicated non-linear equations. Because the full $N$-body Schrödinger equation is, at present, virtually inaccessible to computer calculation while the HF equation, especially in the spherical approximation [6], is ideal for computer iterative solution, the HF equations are extensively used in quantum chemistry [27].

Before our work, the only existing theorems were for the Hartree equation (5) as follows: Reeken [26] considered the restricted Hartree equations for Helium, i.e. he considered (5) with $k=1, z_{1}=2$ and the additional restriction $u_{1}=u_{2}$. He found a solution for this case with $u_{i} \geqq 0$ pointwise; his method works for any $z>1$. Independently Gustafson and Sather [12] found solutions for the restricted two electron problems for sufficiently large $z$ (they state their results for $z=2$ but with $\left\|u_{i}\right\|$ sufficiently small rather than 1 . Since we insist on the normalization condition $\left\|u_{i}\right\|=1$, we scale coordinates to translate their result into a large $z,\left\|u_{i}\right\|=1$ result). These authors all use a bifurcation analysis further discussed in Stuart [32], and depend on the fact that they seek spherically symmetric solutions so that methods of ordinary differential equations are available. Properties of their solutions are further discussed in $[3,4]$. Relations between the restricted Hartree two electron problem and the unrestricted problem appear to present some interesting mathematical phenomena and we hope to return to them in a future publication.

Using a Schauder-Tychonoff theorem, Wolkowisky [34] found ground state and excited solutions of the Hartree equation in the spherical approximation (see e.g. Bethe-Jackiw [6] for a discussion of the approximation). 
All these authors attack the equations directly as fixed point equations in some sense. The reason we are able to go further is that we exploit the form of the equations as gradient maps, i.e. as Euler-Lagrange equations and directly attempt to find solutions by finding minimizing $u$ 's for $\mathscr{E}_{\mathrm{H}}$ and $\mathscr{E}_{\mathrm{HF}}$. (This method has already been used successfully in [23] to find solutions of another of the non-linear equations of atomic physics: the Thomas-Fermi [9,33] model.)

These results for the $\mathrm{H}$ and $\mathrm{HF}$ equations, which we give in $\S 2$ were announced in [22] and sketched in [19]; seemingly unaware of our work, Bader [2] has recently presented a similar method to obtain similar results for the $\mathrm{H}$ (but not $\mathrm{HF}$ ) equations. We note that prior to our work, solutions of the HF equations for a class of potentials excluding Coulomb potentials were found by Fonte et al. [11]. Recently, several authors $[7,8]$ have proved existence of the time-dependent HF equations.

In $\S 3$, we establish various "regularity" properties of any $u$ 's (not necessarily minimizing ones), which solve the $\mathrm{H}$ and $\mathrm{HF}$ equation. Among these is the exponential falloff of the $u$ 's announced in [22]; after our announcement, similar results for the $\mathrm{H}$ equations were obtained by [5].

In $\S 4$, we repeat the remark already made in [23] that our proof that HF theory is "exact" in the $Z \rightarrow \infty$ limit implies the same result for HF theory.

\section{§2. Solutions of the $H$ and HF Equations}

While one could present the existence theory for the $\mathrm{H}$ and $\mathrm{HF}$ equations as two cases of one general result, we present the two theories in sequence to illustrate the extra difficulties in the HF case. The basic strategy is (cf. [23]) to introduce a weak topology on the trial functions in which the trial functions are precompact and then to prove that the functional one wishes to minimize is lower semicontinuous. This establishes that the functional is minimized at some point in the closure of the trial functions. In many cases, additional arguments are then available to prove that the minimizing point belongs to the original trial functions rather than merely to the closure.

Theorem 2.1 (H Theory). Fix $N, k ; z_{1}, \ldots, z_{k}, R_{1}, \ldots, R_{k}$. There exist functions $u_{1}, \ldots, u_{N} \in L^{2}\left(\mathbb{R}^{3} ; \mathbb{C}^{2}\right)$ with $u_{i} \in Q(-\Delta)$, the quadratic form domain of $-\Delta$, such that the $u_{i}$ minimize

$$
\begin{aligned}
\tilde{\mathscr{E}}_{\mathrm{H}}\left(u_{1}, \ldots, u_{N}\right)= & \sum_{i=1}^{N}\left(u_{i},(-\Delta+V) u_{i}\right) \\
& +\sum_{i<j} \int\left|u_{i}(x)\right|^{2}\left|u_{j}(y)\right|^{2}|x-y|^{-1} d^{3} x d^{3} y
\end{aligned}
$$

with the subsidiary conditions, $u_{i} \in Q(-\Delta)$ and

$$
\left\|u_{i}\right\| \leqq 1 \text {. }
$$

The $u_{i}$ 's satisfy (5a) with the additional condition, that for each $i$, either $\varepsilon_{i} \leqq 0$ or $u_{i}=0$. In either event $\varepsilon_{i}=\inf \operatorname{spec}\left(\tilde{h}_{i}\right)$ and if $\varepsilon_{i}<0,\left\|u_{i}\right\|=1$. If, moreover, $N<Z+1$, then all $\varepsilon_{i}<0$ and each $\left\|u_{i}\right\|=1$. In all cases

$$
E_{\mathrm{H}} \equiv \min \left\{\tilde{\mathscr{E}}_{\mathrm{H}}\left(u_{1}, \ldots, u_{N}\right) \mid\left\|u_{i}\right\| \leqq 1, u_{i} \in Q(-\Delta)\right\}
$$

is finite. 
Remark. We have introduced the function $\tilde{\mathscr{E}}_{\mathrm{H}}$ which agrees with $\mathscr{E}_{\mathrm{H}}$ only when all $\left\|u_{i}\right\|=1$. Theorem 2.1 says that $\tilde{\mathscr{E}}_{\mathrm{H}}$ always has a minimum if we only impose $\left\|u_{i}\right\| \leqq 1$. When the minimum of $\tilde{\mathscr{E}}_{\mathrm{H}}$ occurs for $\left\|u_{i}\right\|=1$, all $i$, as we assert it does if $Z+1>N$, then, of course, these $u_{i}$ also minimize $\mathscr{E}_{\mathrm{H}}$ subject to $\left\|u_{i}\right\|=1$.

Proof. By a well-known result of Kato [18], for any $\varepsilon>0$,

$$
(u, V u) \leqq \varepsilon(u,-\Delta u)+C_{\varepsilon}(u, u)
$$

from which it follows that

$$
E_{\mathrm{H}}=\inf \left\{\tilde{\mathscr{E}}_{\mathrm{H}}\left(u_{1}, \ldots, u_{N}\right) \mid\left\|u_{i}\right\| \leqq 1, u_{i} \in Q(-\Delta)\right\}
$$

is finite and that for some $K$ :

$$
\tilde{\mathscr{E}}_{\mathrm{H}}\left(u_{1}, \ldots, u_{N}\right) \leqq E_{H}+1 ;\left\|u_{j}\right\| \leqq 1 \Rightarrow\left\|\nabla u_{i}\right\| \leqq K .
$$

Now pick sets $u_{i}^{(n)}, 1 \leqq i \leqq N, n=1, \ldots$ so that $\tilde{\mathscr{E}}_{\mathrm{H}}\left(u_{i}^{(n)}\right) \leqq E_{\mathrm{H}}+1 / n$. By (9), the $u_{i}^{(n)}$ 's lie in a fixed ball in the Sobolev space [1], $H^{1}=\left\{u\left|\left\||u \|| \equiv\left(\|u\|^{2}+\|\nabla u\|^{2}\right)^{1 / 2}<\infty\right\}\right.\right.$. Thus, by the Banach-Alaoglu theorem, there exists a subsequence such that $u_{i}^{(n)} \rightarrow u_{i}^{(\infty)}$ in the weak-H $\mathrm{H}^{1}$ topology. Clearly $\left\|u_{i}^{(\infty)}\right\| \leqq 1$. We claim that $\tilde{\mathscr{E}}_{\mathrm{H}}\left(u_{i}^{(\infty)}\right)$ $\leqq \underline{\lim } \mathscr{E}_{\mathrm{H}}\left(u_{i}^{(n)}\right)=E_{\mathrm{H}}$, whence it follows that the $u_{i}^{(\infty)}$ minimize $\tilde{\mathscr{E}}_{\mathrm{H}}$. Positive definite quadratic forms are always non-increasing under weak-limits (see e.g. [23]) so that

$$
\begin{aligned}
& \left(u_{i}^{(\infty)},-\Delta u_{i}^{(\infty)}\right) \leqq \underline{\lim }\left(u_{i}^{(n)},-\Delta u_{i}^{(n)}\right) \\
& \left(u_{i}^{(\infty)} u_{j}^{(\infty)},\left|x_{i}-x_{j}\right|^{-1} u_{i}^{(\infty)} u_{j}^{(\infty)}\right) \leqq \underline{\lim }\left(u_{i}^{(n)} u_{j}^{(n)},\left|x_{i}-x_{j}\right|^{-1} u_{i}^{(n)} u_{j}^{(n)}\right)
\end{aligned}
$$

since $u_{i}^{(n)} u_{j}^{(n)} \stackrel{w}{\longrightarrow} u_{i}^{(\infty)} u_{j}^{(\infty)}$ in $L^{2}\left(R^{6}\right)$. Finally, because $[18,25] V$ is relatively $-\Delta$ form compact [i.e. $(\Delta+1)^{-1 / 2} V(-\Delta+1)^{-1 / 2}$ is compact] $\left(u_{i}^{(n)}, V u_{i}^{(n)}\right) \rightarrow\left(u_{i}^{(\infty)}, V u_{i}^{(\infty)}\right)$. It follows that $\underline{\lim } \tilde{\mathscr{E}}_{\mathrm{H}}\left(u_{i}^{(n)}\right) \geqq \tilde{\mathscr{E}}_{\mathrm{H}}\left(u_{i}^{(\infty)}\right)$. Henceforth, $u_{i}$ is used to denote this $u_{i}^{(\infty)}$.

To see that the $u_{i}$ 's satisfy (5a), fix $u_{1}, \ldots, u_{i-1}, u_{i+1}, \ldots, u_{N}$ and let

$$
\begin{aligned}
f(u) & =\tilde{\mathscr{E}}_{\mathrm{H}}\left(u_{1}, \ldots, u_{i-1}, u, u_{i+1}, \ldots, u_{N}\right) \\
& =\mathrm{const}+\left(u, \tilde{h}_{i} u\right) .
\end{aligned}
$$

Since $f(u)$ is minimized by $u=u_{i}$ subject to $\|u\| \leqq 1$, we conclude that either $\tilde{h}_{i} \geqq 0, u_{i}$ $=0$, or $\tilde{h}_{i} u_{i}=\varepsilon_{i} u_{i}$ with $\varepsilon_{i}=\inf \operatorname{spec} \tilde{h}_{i} \leqq 0$.

Now suppose that $N<Z+1$. Let $v$ be a spherically symmetric function on $\mathbb{R}^{3}$. Then $\left(v, \tilde{h}_{i} v\right)=(v,-\Delta v)+(v, K v)$, where

$$
K(r)=-\sum_{j=1}^{k} z_{j}\left(\max \left(r,\left|R_{j}\right|\right)\right)^{-1}+\sum_{j \neq i} \int\left|u_{j}(k)\right|^{2}(\max (x, r))^{-1} d x .
$$

Since $\left\|u_{j}\right\| \leqq 1$, we have that

$$
K(r) \leqq-[Z-(N-1)]|r|^{-1} \text { when } \quad r>\max \left(\left|R_{j}\right|\right) .
$$

It is easy to see (use explicit hydrogenic wave functions, or a scaling argument [28]), that $\left(v, h_{i} v\right)<0$ for suitable $v$ 's. It follows that $\varepsilon_{i}<0$ so that $\left\|u_{i}\right\|=1$.

Remark. 1) In particular, in the neutral case $\sum z_{j}=N$, a solution of the $H$ equation exists.

2) Notice that no assertion is made about uniqueness.

3) In the above proof, we used $\left|x_{i}-x_{i}\right|^{-1} \geqq 0$ pointwise. In distinction, at the 
analogous point in the TF theory we used the fact that $|x|^{-1}$ is positive definite.

4) The above method fails for the Hartree-like Choquard functional $\mathscr{E}(u, v)=\|\nabla u\|^{2}+\|\nabla v\|^{2}-\int|u(x)|^{2}|v(y)|^{2}|x-y|^{-1} d x d y$, because the last term is negative instead of positive. Nevertheless, alternate methods involving rearrangement inequalities can be used to prove that minimizing $u$ and $v$ exist, see Lieb [21].

5) Since the $u_{i}$ 's are ground states of $h_{i}$, they are pointwise positive [25].

To prove the existence of solutions of the HF equation, we must extend $\mathscr{E}_{\mathrm{HF}}$ in a manner analogous to (8); we define:

$$
\tilde{\mathscr{E}}_{\mathrm{HF}}\left(u_{1}, \ldots, u_{N}\right)=\sum_{i=1}^{N}\left(u_{i},(-\Delta+V) u_{i}\right)+\sum_{1 \leqq i<j \leqq N}\left((i j)_{2},\left|x_{i}-x_{j}\right|(i j)_{2}\right),
$$

where

$$
(i j)_{2}\left(x_{i}, x_{j}\right)=2^{-1 / 2}\left[u_{i}\left(x_{i}\right) u_{j}\left(x_{j}\right)-u_{j}\left(x_{i}\right) u_{i}\left(x_{j}\right)\right] .
$$

For future reference we note that

$$
\begin{aligned}
& \left((i j)_{2},\left|x_{i}-x_{j}\right|^{-1}(i j)_{2}\right)=D_{i j}-E_{i j} \\
& D_{i j}=\int|x-y|^{-2}\left|u_{i}(x)\right|^{2}\left|u_{j}(y)\right|^{2} d x d y \\
& E_{i j}=\int|x-y|^{-1} \overline{\varrho(x)} \varrho(y) d x d y \\
& \varrho(x)=u_{i}(x) \overline{u_{j}(x)} .
\end{aligned}
$$

The critical element in the extension will be to locate the weak closure of $\left\{\left(u_{1}, \ldots, u_{N}\right) \mid\left(u_{i}, u_{j}\right)=\delta_{i j}\right\}$ :

Lemma 2.2. Let $u_{i}^{(n)} \rightarrow u_{i}(i=1, \ldots, N)$ weakly with $\left(u_{i}^{(n)}, u_{j}^{(n)}\right)=\delta_{i j}$. Then $\left(u_{i}, u_{j}\right)=M_{i j}$ is an $N \times N$ matrix with $0 \leqq M \leqq 1$. More generally the conclusion remains true if the weaker hypothesis $\left(u_{i}^{(n)}, u_{j}^{(n)}\right)=M_{i j}^{(n)}$ with $0 \leqq M^{(n)} \leqq 1$ is imposed.

Remark. The point is that it is easy to see that every $\left(u_{1}, \ldots, u_{N}\right)$ with $M_{i j}$ obeying 0 $\leqq M \leqq 1$ arises as a weak limit of orthonormal $N$-tuples. Since we do not need this below, we do not give the easy proof of this converse which is based on diagonalizing $M$.

Proof. Let $z \in \mathbb{C}^{N}$. Then $(z, M z) \equiv \sum \bar{z}_{i} M_{i j} z_{j}=(u(z), u(z))=\left(w-\lim u^{(n)}(z), w-\lim u^{(n)}(z)\right)$ $\leqq \sum\left|z_{i}\right|^{2}$ where $u^{(n)}(z)=\sum z_{i} u_{i}^{(n)}$. The last inequality follows from the fact that balls are weakly closed and the calculation $\left(u^{(n)}(z), u^{(n)}(z)\right)=\sum\left|z_{i}\right|^{2}$. Thus $M \leqq 1 . M \geqq 0$ is trivial.

We will also need the elementary observation:

Lemma 2.3. Let $\tilde{u}_{i}=\sum_{j} a_{i j} u_{j}$ where $A=\left\{a_{i j}\right\}_{1 \leqq i, j \leqq N}$ is a unitary $N \times N$ matrix then $\tilde{\mathscr{E}}_{\mathrm{HF}}\left(\tilde{u}_{i}\right)=\tilde{\mathscr{E}}_{\mathrm{HF}}\left(u_{i}\right)$.

Proof. Let $K_{i j}=\left(u_{i},(-\Delta+V) u_{j}\right), \quad \tilde{K}_{i j}=\left(\tilde{u}_{i},(-\Delta+V) \tilde{u}_{j}\right), \quad R_{i_{1} i_{2} j_{1} j_{2}}=\left(\left(i_{1} j_{2}\right)_{2}\right.$, $\left.|x-y|^{-1}\left(j_{1} j_{2}\right)_{2}\right)$, etc. Then $\tilde{K}=A^{*} K A \quad$ so $\sum \tilde{K}_{i i}=\operatorname{Tr}(\tilde{K})=\operatorname{Tr}(K)=\sum K_{i i}$. Similarly, by taking traces on the antisymmetric tensor product of $\mathbb{C}^{N}$ with itself

$$
\sum_{i<j} \tilde{R}_{i j, i j}=\sum_{i<j} R_{i j, i j}
$$


Theorem 2.4 (HF Theory). Fix $N, k ; z_{1}, \ldots, z_{k}, R_{1}, \ldots, R_{k}$. There exist functions $u_{1}, \ldots, u_{N} \in L^{2}\left(\mathbb{R}^{3} ; \mathbb{C}^{2}\right)$ such that $u_{i} \in Q(-\Delta)$, the quadratic form domain of $-\Delta$, and such that the $u_{i}$ minimize $\tilde{\mathscr{E}}_{\mathrm{HF}}$ (given by (10)) with the subsidiary condition.

$$
M_{i j} \equiv\left(u_{i}, u_{j}\right) \quad \text { obeys } \quad 0 \leqq M \leqq 1 .
$$

Moreover, the u's obey $\left(u_{i}, u_{j}\right)=\lambda_{i} \delta_{i j}$ and satisfy the HF equations (7) with the additional condition that either $\varepsilon_{i} \leqq 0$ or $u_{i}=0$ for each $i . \varepsilon_{i}, \ldots, \varepsilon_{N}$ are the $N$ lowest points of the spectrum of $h$ and if $\varepsilon_{i}<0, \lambda_{i}=1$. If moreover, $N<Z+1$, then all $\varepsilon_{i}<0$ and $\left(u_{i}, u_{j}\right)=\delta_{i j}$.

Proof. By mimicking the proof of Theorem 2.1, we find $u_{i}^{(\infty)}$ obeying $0 \leqq M^{(\infty)} \leqq 1$ which minimize $\tilde{\mathscr{E}}_{\mathrm{HF}}$. In this proof, we use Lemma 2.2 to be sure that $0 \leqq M^{(\infty)} \leqq 1$ and the fact that $(\mathrm{ij})^{(n)} \rightarrow(\mathrm{ij})^{(\infty)}$ if $u_{i}^{(n)} \rightarrow u_{i}^{(\infty)}$.

Choose a unitary $N \times N$ matrix $A$ so that $A^{*} M A$ is diagonal and let $u_{i}=\sum_{j} a_{i j} u_{j}^{(\infty)}$. By Lemma 2.3, $\left\{u_{i}\right\}$ minimizes $\tilde{\mathscr{E}}_{\mathrm{HF}}$ also, and clearly $\left(u_{i}, u_{j}\right)=\lambda_{i} \delta_{i j}$. Now $F(u) \equiv \tilde{\mathscr{E}}_{\mathrm{HF}}\left(u_{1}, \ldots, u_{i-1}, u, u_{i+1}, \ldots, u_{\mathrm{N}}\right)=\mathrm{const}+(u, h u)$ so since $u=u_{i}$ minimizes $F(u)$ subject to $\left(u, u_{j}\right)=0(j \neq i),(u, u) \leqq 1, u_{i}$ must be a linear combination of the $N$ smallest eigenvectors of $h$ with only eigenvalues $\leqq 0$ allowed. Since each $u_{i}$ has this property, by further unitary change, the $u_{i}$ 's can be made to obey $h u_{i}=\varepsilon_{i} u_{i}$.

To complete the proof we need only show that if $N-1<Z$, then $h$ has $N$ points of its spectrum below zero, i.e. $\operatorname{dim} P_{(-\infty, 0)}(h) \geqq N$. Now write $h=h_{0}-K_{\Psi}$ with $K_{\Psi}$ given by (7d). By the positive definitness of $|x|^{-1}, K_{\Psi}$ is a positive operator, so we need only show that $h_{0}$ has $N$ negative eigenvalues. This follows, as in the proof of Theorem 2.1 by considering spherically symmetric trial functions.

As already remarked in [22], the above method also yields solutions of modified HF equations in which we restrict the $u$ 's to lie in certain sets with suitable properties. Because of the spin independence of the assumed Hamiltonian, $H$, we can obtain several solutions of the actual HF equation by taking each $u_{i}$ to be a product of a space and spin function, for example, and demanding a particular symmetry of the spinor functions. We emphasize that the "true" minimum of Theorem 2.4 is not known to come from a set of $u$ 's which are product functions. An additional restriction which is often made and for which our method applies, is to demand the spatial functions be real.

\section{§3. Properties of Solutions}

Theorem 3.1. The solutions of the $\mathrm{H}$ equation (5) constructed in Theorem 2.1 obey:

a) The $u_{i}$ are globally Lipschitz and lie in $D\left(\tilde{h}_{i}\right)=D(-\Delta)$.

b) Away from the points $r=R_{j}$, the $u_{i}$ are infinitely differentiable.

c) Exponential falloff: For any $\alpha<\left|\varepsilon_{i}\right|^{1 / 2}$ there exists $a C_{\alpha}$ so that

$$
\left|u_{i}(x)\right| \leqq C_{\alpha} \exp (-\alpha|x|) \text {. }
$$

Proof. $\tilde{h}_{i}=-\Delta+\tilde{V}_{i}$, with $\tilde{V}_{i}=V+R_{\psi}^{i}$. Since $u_{j} \in L^{2} \cap Q(-\Delta)$, by a Sobolov estimate [31] (for a simple discussion of this point, see [20]) it follows that $u_{j}^{2} \in L^{1} \cap L^{3 / 2-\varepsilon}$, so by Young's inequality, $R_{\Psi}^{i} \in L^{\infty}$. Thus $D\left(\tilde{h}_{i}\right)=D(-\Delta)$. Since $u_{i} \in D\left(\tilde{h}_{i}\right)$, we have $u_{i}$ 
continuous so that $R_{\Psi}^{i}$ is continuous. a) Now follows by a result of Kato [17] and c) by a result of Simon [29] (both specialized to the two-body case; see the original papers for other references including earlier results for the two body case). b) Follows by a bootstrap argument reminiscent of our argument in [23]: We exploit the following facts (see e.g. [24], Section IX.6):

a) Let $\Omega$ be a bounded open set. If $u \in L^{2}(\Omega) ;-\Delta u=W u$ and $W \in C^{k}(\Omega)$, then $D^{\alpha} u \in L^{2}$ for all multiindices $\alpha$ with $|\alpha| \leqq k+2$.

b) If $D^{\alpha} u \in L^{2}(\Omega)$ for all $\alpha$, then $u$ is $C^{\infty}$ on $\Omega$.

The additional fact which we need is that if $u \in L^{2}\left(\mathbb{R}^{3}\right)$ and if $D^{\alpha} u \in L^{2}(\Omega),|\alpha| \leqq m$, then $g(x)=\int|u(y)|^{2}|x-y|^{-1} d y$ is $C^{m}$ on $\Omega$. This follows by writing $|x|^{-1}=\varphi_{1}+\varphi_{2}$ with $\varphi_{2} \in C^{\infty}$ with support outside a ball of radius $\varepsilon / 2$ and $\varphi_{1}$ supported in a ball of radius $\varepsilon$. Then $g(x)=\int|u(y)|^{2} \varphi_{1}(x-y) d y+\int|u(y)|^{2} \varphi_{2}(x-y) d y \equiv g_{1}+g_{2}$. The $g_{2}$ term is $C^{\infty}$ on all of $\mathbb{R}^{3}$. The $g_{1}$ term is easily seen to be $C^{m}$ on those $x$ such that $\{y|| x$ $-y \mid<\varepsilon\} \subset \Omega$.

With a), b), and the above fact, $u_{j}$ is $C^{\infty}$ away from the $R_{j}$ by an obvious inductive argument.

At first sight, the methods of Theorem 3.1 appear to be inapplicable to the HF case because of the non-local term. However, an elementary trick allows one to write the HF equations in local form; namely we consider the operator $A$ on $\bigoplus_{j=1}^{N} L^{2}\left(R^{3} ; \mathbb{C}^{2}\right)$ given by :

$$
A_{i j}=\delta_{i j}\left(-\Delta+V(x)+R(x)-\varepsilon_{i}\right)+Q_{i j}(x)
$$

with

$$
Q_{i j}(x)=-\int|x-y|^{-1} \overline{u_{j}(y)} u_{i}(y) d y .
$$

Then $A \Psi=0$ where $\Psi$ is the vector with $\Psi_{i}(x)=u_{i}(x)$. With this remark, the following can be proven by following the proof of Theorem 3.1:

Theorem 3.2. The solutions of the HF equation (7) constructed in Theorem 2.4 obey:

a) The $u_{i}$ are globally Lipschitz and lie in $\mathscr{D}(h)=D(-\Delta)$.

b) Away from the points $r=R_{j}$, the $u_{i}$ are $C^{\infty}$.

c) Let $k_{0}=\min _{i}\left|\varepsilon_{i}\right|^{1 / 2}$. Then for any $\alpha<k_{0}$ :

$\left|u_{i}(x)\right| \leqq C_{\alpha} \exp (-\alpha|x|), \quad$ all $i$.

Remarks. 1) The common exponential rate of falloff which was obtained comes about because we have written the HF equation as a single multicomponent equation. However, it is evident that barring some miraculous cancellation, the $u_{i}$ 's should have the same rate of falloff because in the HF equations $\left[(-\Delta+V) u_{i}\right](x)$ is a sum of terms containing all the $u_{j}(x)$ 's in them. After making this remark in [22], we learned that it had already been made in the chemical physics literature [13].

2) By following the method of Kato [17] (or an alternative of Jensen [15]) one can give the precise singularity in the first derivatives of the $u_{i}$ at the points $R_{j}$.

3) We believe that the $u_{i}$ 's are real analytic away from the $R_{j}$ 's.

4) In both Theorems 3.1 and 3.2, only the form of the equations and $u_{i} \in Q(-\Delta) \cap L^{2}$ is used. Any solutions satisfying this $Q(-\Delta)$ condition will obey the conclusions of the theorems. 


\section{$\S$ A. Connection with the Quamtunm Theory}

We make explicit a remark of ours in [23]:

Theorem $\mathbb{1}_{0} \mathbb{1}$. Let $E_{N}^{Q}\left(z_{i}, R_{i}\right)$ be given by (3) and define

$$
E_{N}^{\mathrm{HF}}\left(z_{i}, R_{i}\right) \equiv \inf \left\{(\Psi, H \Psi) \mid \Psi \in \mathscr{D}_{\text {phys }} ;\|\Psi\|=1 ; \Psi \text { a Slater determinant }\right\} .
$$

Let $R_{i}, z_{i}$ be $N$ dependent in the following manner:
a) $z_{i} / N \rightarrow \lambda_{i}$.
b) $R_{1}=0 ; R_{j} N^{-1 / 3} \rightarrow r_{j}$ or to $\infty$.

Then

$$
\lim _{N \rightarrow \infty} E_{N}^{\mathrm{HF}}\left(z_{i}, R_{i}\right) / E_{N}^{Q}\left(z_{i}, R_{i}\right)=1 .
$$

Proof. $E_{N}^{Q} \leqq E_{N}^{\mathrm{HF}}<0$ by the variational principle so clearly the $\overline{\mathrm{im}}$ is $\leqq 1$. In our proof that $T F$ theory is asymptotically correct ( $\$$ III of [23]) we constructed an explicit Slater determinant so that as $N \rightarrow \infty,(\Psi, H \Psi) / E_{N}^{T F} \rightarrow 1$ (where $E^{T F}$ is the Thomas-Fermi energy). Since $E_{N}^{Q} / E_{N}^{T F} \rightarrow 1$ by [23], and $E_{N}^{\mathrm{HF}} \leqq(\Psi, H \Psi)$, the $\underline{\mathrm{lim}} \geqq 1$.

Remarks. 1) As explained in [23], we expect $E_{N}^{\mathrm{HF}}-E_{N}^{Q}=o\left(N^{5 / 3}\right)$ and $E_{N}^{Q}=a N^{7 / 3}$ $+b N^{2}+c N^{5 / 3}+o\left(N^{5 / 3}\right)$. The proof of these facts seems to us to be an important problem in understanding the bulk properties of large $Z$ atoms and molecules. All we were able to obtain rigorously is the leading term $a N^{7 / 3}$. In [23], a conjecture, due to Scott, is made about the next term $b N^{2}$ (see [20] for more details).

2) We emphasize that Theorem 4.1 is only a limit theorem about total binding energy. It is physically more important to prove that HF theory gives asymptotically correct ionization energies.

\section{References}

1. Adams, R.: Sobolev spaces. New York: Academic Press 1976

2. Bader,P.: Méthode variationelle pour l'équation de Hartree. E.P.F. Lausanne Thesis

3. Bazley, N., Seydel, R.: Existence and bounds for critical energies of the Hartree operator. Chem. Phys. Letters 24, 128-132 (1974)

4. Behling, R., Bongers, A., Kuper, T.: Upper and lower bounds to critical values of the Hartree operator. University of Köln (preprint)

5. Benci,V., Fortunato,D., Zirilli,F.: Exponential decay and regularity properties of the Hartree approximation to the bound state wavefunctions of the helium atom. J. Math. Phys. 17, 1154 -1155 (1976)

6. Bethe,H., Jackiw, R. : Intermediate quantum mechanics. New York: Benjamin 1969

7. Bove, A., DaPrato, G., Fano, G.: An existence proof for the Hartree-Fock time dependent problem with bounded two-body interaction. Commun. math. Phys. 37, 183-192 (1974)

8. Chadam,J.M., Glassey,R.T.: Global existence of solutions to the Cauchy problem for timedependent Hartree equations. J. Math. Phys. 16, 1122-1130 (1975)

9. Fermi,E.: Un metodo statistico per la determinazione di alcune priorietà dell atome. Rend. Acad. Nat. Lincei 6, 602-607 (1927)

10. Fock, V. : Näherungsmethode zur Lösing des quantenmechanischen Mehrkörperproblems. Z. Phys. 61, 126--148 (1930)

11. Fonte, G., Mignani, R., Schiffrer, G. : Solution of the Hartree-Fock equations. Commun. math. Phys. $33,293-304$ (1973) 
12. Gustafson, K., Sather,D.: Branching analysis of the Hartree equations. Rend. di Mat. 4, 723-734 (1971)

13. Handy, N.C., Marron, M.T., Silverstom, H.J. : Long range behavior of Hartree-Fock orbitals. Phys. Rev. 180, 45-47 (1969)

14. Hartree,D.: The wave mechanics of an atom with a non-coulomb central field. Part I. Theory and methods. Proc. Comb. Phil. Soc. 24, 89-132 (1928)

15. Jensen, R.: Princeton University Senior Thesis, 1976

16. Kato, T.: Fundamental properties of Hamiltonian operator of Schrödinger type. Trans. Am. Math. Soc. 70, 195-211 (1951)

17. Kato, T.: On the eigenfunctions of many particle systems in quantum mechanics. Comm. Pure Appl. Math. 10, 151-177 (1957)

18. Kato,T.: Perturbation theory for linear operators. Berlin-Heidelberg-New York: Springer 1966

19. Lieb,E.H.: Thomas-Fermi and Hartree-Fock theory, Proc. 1974 International Congress Mathematicians, Vol. II, pp. 383-386

20. Lieb,E.H.: The stability of matter. Rev. Mod. Phys. 48, 553-569 (1976)

21. Lieb,E.H.: Existence and uniqueness of minimizing solutions of Choquard's non-linear equation. Stud. Appl. Math. (in press)

22. Lieb, E.H., Simon, B.: On solutions to the Hartree Fock problem for atoms and molecules. J. Chem. Phys. 61, 735-736 (1974)

23. Lieb,E.H., Simon, B.: The Thomas-Fermi theory of atoms, molecules, and solids. Adv. Math. 23, $22-116(1977)$

24. Reed, M., Simon, B. : Methods of modern mathematical physics. II. Fourier analysis, self-adjointness. New York: Academic Press 1975

25. Reed,M., Simon, B.: Methods of modern mathematical physics. IV. Analysis of operators. New York: Academic Press 1977

26. Reeken,M.: General theorem on bifurcation and its application to the Hartree equation of the helium atom. J. Math. Phys. 11, 2505-2512 (1970)

27. SchaeferIII,H.F.: The electronic structure of atoms and molecules. Reading: Addison Wesley 1972

28. Simon, B.: On the infinitude vs. finiteness of the number of bound states of an $\mathrm{N}$-body quantum system. Helv. Phys. Acta 43, 607-630 (1970)

29. Simon, B.: Pointwise bounds on eigenfunctions and wave packets in $N$-body quantum systems. I. Proc. Am. Math. Soc. 42, 395-401 (1974)

30. Slater,J.C.: A note on Hartree's method. Phys. Rev. 35, 210-211 (1930)

31. Stein,E.: Singular integrals and differentiability properties of functions. Princeton: University Press 1970

32. Stuart, C.: Existence theory for the Hartree equation. Arch. Rat. Mech. Anal. 51, 60-69 (1973)

33. Thomas, L.H.: The calculation of atomic fields. Proc. Comb. Phil. Soc. 23, 542-548 (1927)

34. Wolkowisky, J.: Existence of solutions of the Hartree equations for $N$ electrons. An application of the Schauder-Tychonoff theorem. Ind. Univ. Math. Journ. 22, 551-558 (1972)

Communicated by J. Glimm

Received December 17, 1976 